\title{
PHYSICAL PROPERTIES OF SPECKLES
}

\author{
H. J. Tiziani
}

Wild Heerbrugg Ltd.

Heerbrugg

Switzerland

The speckle phenomenon has long been familiar, but only the introduction of lasers has brought a deeper understanding and many new applications. Laser speckles appear whenever an optically rough surface is illuminated with highly coherent light. The roughness of the surface needs to be of the order of the wavelength of the light used, or coarser. The optical wave originating from any moderately distant point consists of many coherent wavelets, each arising from a different element of the surface. The optical path difference between these various wavelets may differ by several wavelengths. In addition, the waves scattered from an optically rough surface have not only random phases but also random real amplitudes [2.1]. The interference of the dephased, but coherent, secondary spherical wavelets results in the granular pattern of intensity that is termed speckle. Laue [2.2] derived many basic results that have direct relevance to speckling, and statistical phenomena entirely analogous to laser speckle are found in radio wave propagation [2.3].

Speckles appear everywhere in space whenever an optically rough surface is illuminated with highly coherent light. We are, however, mostly interested in the image and Fraunhofer diffraction planes. The random field distribution is stationary in time and is a function of the spatial coordinates. Consider the factors that qualitatively determine the shape of the speckles, and the form of the envelope of the intensity in the diffraction pattern. In Fig. 2.1 a rough object is illuminated by a coherent wave, converging to a focus in the neighborhood of the point $E$. For simplicity, transmitted illumination is shown, but the discussion applies equally well to a reflected wave. The roughness is assumed to be localized on the exit surface of the object. A simple physical picture of what occurs may be obtained by considering the irregularities to comprise a random set of positive and negative lenses. The pencils of rays falling on two such lens elements are indicated in the diagram. The rays marked with double 


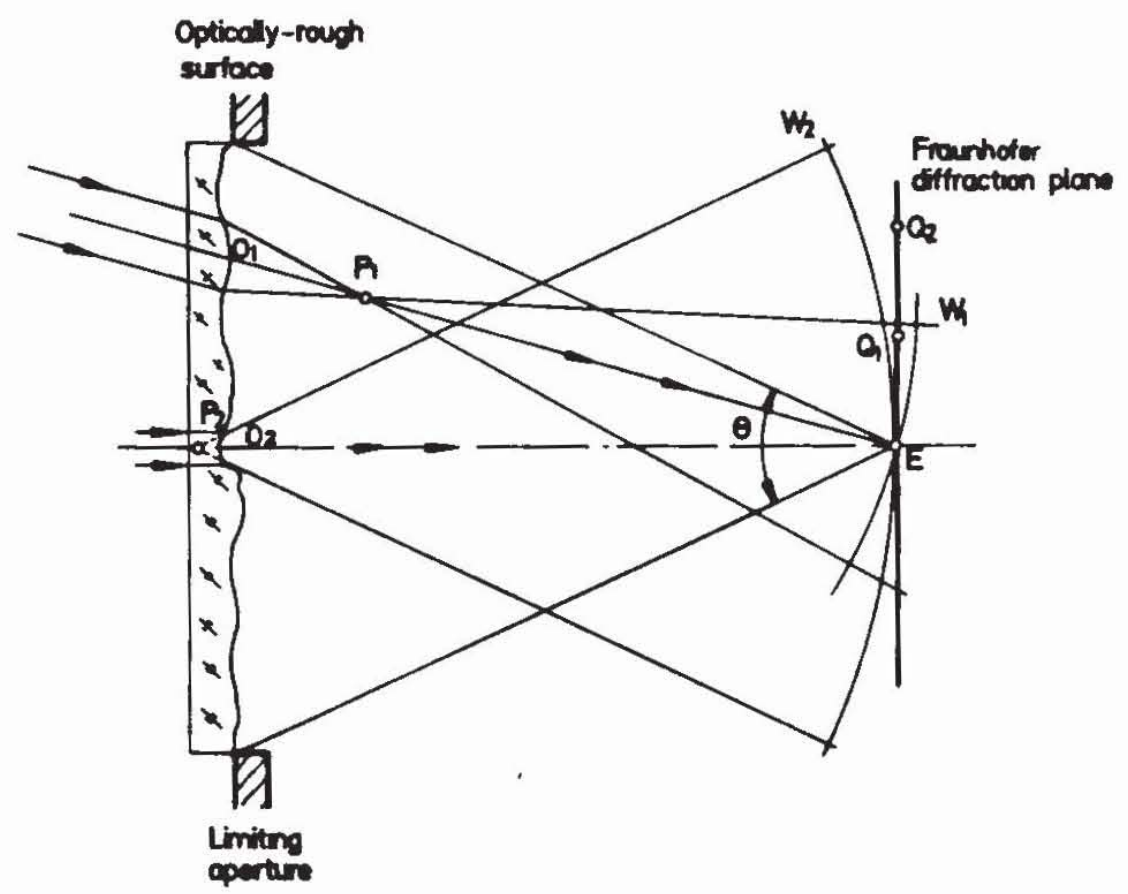

Fig. 2.1 Schematic diagram illustrating the formation of speckling in the Fraunhofer diffraction plane.

arrows pass undeviated to the point $E$. The convex lens element at $O_{1}$ focuses rays at $\boldsymbol{P}_{1}$, from which they will diverge as a relatively narrow pencil, giving a wave $W_{1}$. The concave lens element at $O_{2}$ produces a pencil of rays coming from the virtual image $\boldsymbol{P}_{2}$ which, because of the deeper curvature of the lens element, diverges as a wider-angle pencil, giving the wave $W_{2}$. In the diffraction plane the total disturbance will be the resultant of the mutual interference of the coherent waves produced by all such elements of the object. These waves will have random mutual phases because of the random variation of the optical thickness of the object.

At a point such as $Q_{1}$ there will be light from all elements of the object, whereas only those elements giving wide-angle scattering will send light to a point such as $Q_{2}$ further from the center $E$ of the pattern. Since the maximum intensity that can be produced increases with the number of interfering waves, the envelope of the intensity in the diffraction pattern will be expected to have a greater value at $Q_{1}$ than at $Q_{2}$. Thus, if the size deviation of scattering elements relative to the mean exhibits a Gaussiantype distribution, the envelope of the diffraction pattern will be expected to have a maximum at the center $E$ and to decrease continuously to the edge. The form of this transition curve will depend on the standard deviations of the sizes and curvatures of the elements. For example, if all elements were of the same size and curvature, the scattered waves would have the same angular spread, and there would be a large constant en- 
velope of intensity, with a rapid decrease to zero at the edge. The greater the size range of the surface elements, the greater will be the angular range of scattering produced. In this case there will be a smaller constant region, and a more gradual transition to the edge of the pattern. Figure 2.2 shows the distribution of light in the Fraunhofer diffraction pattern for two ground glass plates having different roughnesses: very coarse ground glass gives a narrow envelope of light distribution (Fig. 2.2A), and fine ground glass gives a broad envelope (Fig. 2.2B). The frequency spectra of the corresponding surface roughnesses are depicted in Figs. 2.2C and 2.2D, respectively.

The detailed structure of individual speckles, however, is determined by the size of the illuminated object field. The smallest detail that can occur in the diffraction pattern is determined by the angular size of the diffracting object as seen from the diffraction plane. The diffraction pattern may be regarded as produced by the coherent superposition of the interference fringes of the waves falling on the plane $E$ taken in pairs. The finest structure in the pattern corresponds to that produced by the waves with the greatest mutual inclination, i.e., to that formed by the elements
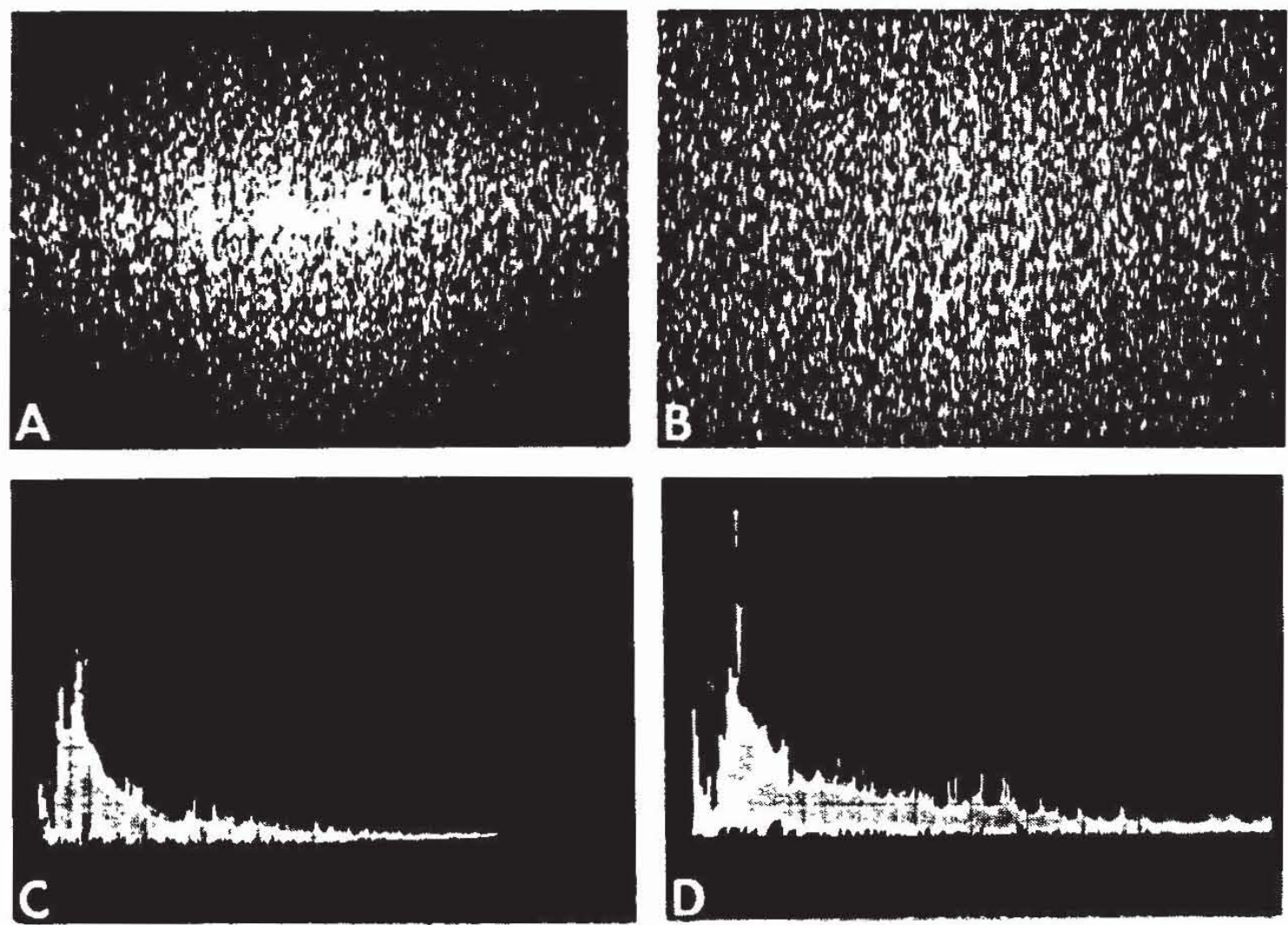

Fig. 2.2 Speckle patterns as recorded in the Fraunhofer diffraction plane for coarse grained (A) and fine grained (B) ground glass plates, together with the frequency spectra ((C) and (D)) of the corresponding surface roughnesses for the coarse and fine grained cases respectively. 
near to the edge in the schematic diagram of Fig. 2.1. Thus, if the object in any azimuth subtends an angle $\theta$ at $E$, the smallest detail in the random pattern will have a width of the order of $\lambda / \theta$ in this azimuth. The scale of the speckling is predominantly of this order. The above simple considerations account for the general form of envelope and smallest detail size in the speckling that is found in practice. Thus, if the exposed area of the object is reduced by a field stop, the envelope of the diffraction pattern is little affected, but the scale of the speckling is increased in inverse proportion to the angular subtense $\theta$ of the object. This is to be expected, provided the area of the object employed is large enough to include a representative sample of its random structure. The above physical considerations serve to establish reasons for some of the general features of the speckled diffraction patterns observed from rough objects. They do not of themselves, however, give information about the expected predominant size and contrast of the speckles. According to some statistical considerations $[2.1,2.4,2.5]$, the contrast depends on the roughness of the surface and the coherence of the illumination and can reach unity, and the predominant size is obtained by finding the autocorrelation function of the diffracted intensity distribution.

However, consider the case of Fraunhofer diffraction, since the unscattered wave is assumed to focus at, or near to, $E$. (In other cases the scattered waves, such as $W_{1}$ and $W_{2}$, will all be laterally displaced relative to each other, and will merely alter the form of the envelope of the diffraction pattern.) In the formation of the image of a rough object using coherent light, the entrance pupil is illuminated by a speckled diffraction pattern. This random illumination then appears in the exit pupil, so that the speckling in the image is determined by considering the exit pupil to act as a rough object. The order of size of speckling in the image is thus determined by the formula $\lambda / \theta_{0}$, where $\theta_{0}$ is the total convergence angle of the image-forming pencil, as illustrated in Fig. 2.3. The speckle size or

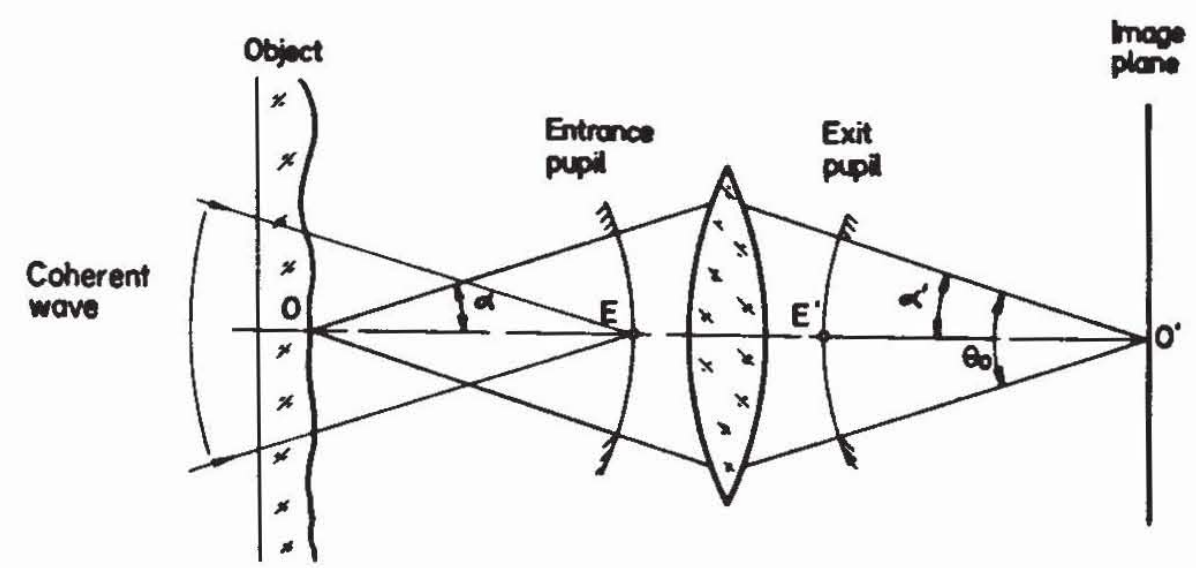

FIG. 2.3 Schematic diagram illustrating the recording of speckling in the image plane. 
shape can be altered by changing the size or shape of the lens aperture. Thus, speckles are no longer regarded merely as a noise element, but as a carrier of information to be used in different applications. This offers a powerful and simple tool for studying surface roughness, for measuring displacement and strain, and for deformation and vibration analysis, all of which are considered in the following chapters.

\section{References}

2.1. H. H. Hopkins and H. J. Tiziani, Speckling in diffraction patterns and optical images formed with the laser, Proc. Int. Symp. Hologr., Besançon, 1970.

2.2. M. Laue, Mathematische Betrachtungen über die Beugungserscheinungen an vielen unregelmässig verstreuten Teilchen, Mitt. Physik. Ges. 18, 90 (1916).

2.3. J. A. Ratcliffe, Some aspects of diffraction theory and their application to the ionosphere, Rep. Progr. Phys. 19, 188 (1956).

2.4. L. I. Goldfischer, Autocorrelation function and power spectral density of laserproduced speckle patterns, J. Opt. Soc. Amer. 55, 247 (1965).

2.5. J. W. Goodman, Statistical properties of laser speckle patterns, in "Laser Speckle and Related Phenomena” (J. C. Dainty, ed.). Springer-Verlag, Berlin and New York, 1975. 